\title{
Research on the Enhancement of Computer Network Robustness Methodology based on the Fuzzy Set Theory
}

\author{
Xiaoqin Song ${ }^{1}$ \\ ${ }^{1}$ Sias International University,Xinzheng,Henan, 451150,China
}

\begin{abstract}
In this paper, we conduct research on the enhancement of computer network robustness methodology based on the fuzzy set theory. The computer network is the distributed in different geographical regions computer with the special external devices connected by communication lines into a large scale and functional system. Protecting the safety of the network is important and urgent. Its topology structure not only can directly reflect the computer support network operation ability strong or weak of the important performance and also can present the network running status. Our proposed method will enhance the current pattern of the network security.
\end{abstract}

Keywords: Network Robustness; Fuzzy Set Theory; Network Topology; Structure Optimization.

\section{INTRODUCTION}

Computer networks refers to the geographical location of different multiple general computers with independent functions and its peripheral equipment, through the communication lines connected, in the management of the network operating system and network communication protocol, realize resources sharing and information transmission of computer system. The computer network is distributed in different geographical regions computer with the special external devices connected by communication lines into a large scale and functional system, so that many computers can easily pass information to each other, such as data resources. This paper mainly discusses the computer network reliability enhance strategy for the relevant departments in improving computer network to provide reference for the further development of stability and reliability. Computer network security status stability analysis is the study of the safety control effect is an important evaluation index. System safety stability includes two kinds of internal stability and external stability. Stability is to point to inside, when disturbance effect on the network system safety rule out after the system's ability to recover, or recover the accuracy of performance.

The computer network reliability is mainly refers to under certain conditions, certain time range, Internet connection and could satisfy the requirement of communication skills. Its topology structure not only can directly reflect the computer support network operation ability strong or weak of the important performance, also can present the network running status for people, in addition, the computer network reliability for future development and the establishment of the structure itself also has a great influence. In the process of computer network operation, once appear problem, is bound to bad influence on people's life and work, severe cases, also can cause economic losses. Computer network is a very complicated system, which is composed of multiple components, only good for reliability and stability of the various parts of the study, enhance the security of the parts, can make whole the stability and security of the computer network to get maximum increase. To this end, it's to the computer network designers put forward higher requirements [1-3].

Network security is a complex project, which involves the technology, equipment, management, use and legislation system, and so on various aspects of factors. Want to well realize information security, we must form a total 
complete set of network information security system, technology, equipment, management, use and factors such as the legislative system of coordinated development, be short of one cannot. The general strategy could be separated into the following sections. (1) The firewall. Firewall is a kind of exclusive hardware, also can be set up a set of software on common hardware. Logically, the firewall is a separator, the limiter and analyzer, main effect is when the communication between two or more network, can have the scale of the access control of the firewall and filtering information. (2) Network anti-virus technology. Can computer virus is a program that can also be a executable code, can destroy the normal operation of the computer, so can't normal use, and even the whole operating system or hard disk is damaged, they just like biological viruses, can also replicate and spread in great quantities, cause a large area of computer network security problems. Establish a comprehensive, multi-level virus protection system, and in a timely manner system upgrades, to ensure that the network from virus. (3) Data encryption. Data encryption technology refers to the data transmission, the information some mixed addition, recoding, so as to achieve the function of the hidden information, make illegal users cannot read the information content, effectively protect the information system and data security, network security is one of the core technology. (4) Access control technology. Access control technology is by setting the access permissions to restrict access to the subject of access object access, consciously or unconsciously to prevent users' access to data. (5) Disaster technology. The information age, the data is more and more prominent, the security of data but also affect the survival of an organization development key.

In this paper, we conduct research on the enhancement of computer network robustness methodology based on the fuzzy set theory. The figure one shows the general logistics.

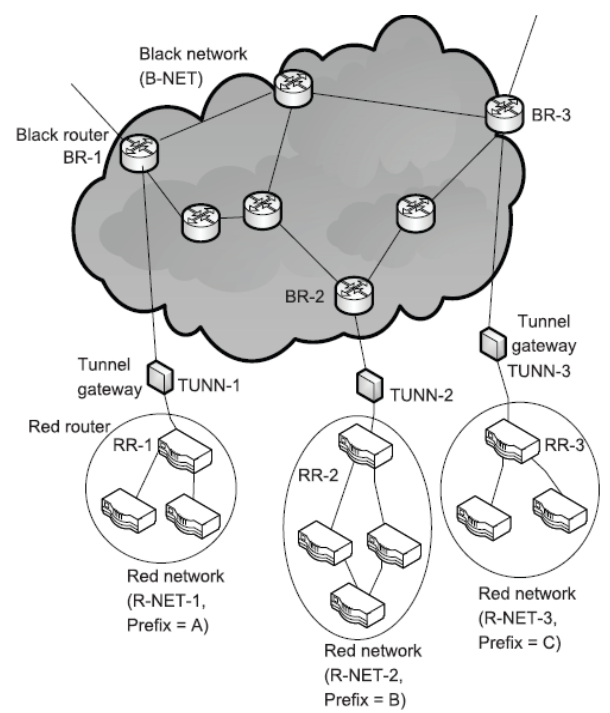

Fig. 1The General Logistics of the Enhancement of Computer Network Robustness

\section{The Proposed Approach and Methodology}

The Fuzzy Set Theory. Fuzzy comprehensive evaluation method is affected by many factors make a comprehensive and objective evaluation of things a way of multifactor decision-making. Using the fuzzy comprehensive evaluation method to calculate correlation process is: to determine the factors and evaluation sets, calculate the membership degree, determine the weight, calculating relevancy. Clustering is given using mathematical method research and processing data object, the classification and all kinds of personal relationship between the degree, is a tool for analysis of a given data object. Feature vector to be established between data sets. According to the similar data in feature vector between narrow close to each other and the thought of different categories of data it is far away, through clustering algorithm to detect the abnormal data. According to the degree of similarity between data points data set can be divided into $\mathrm{k}$ is not the same kind of process is called clustering. First of all, in the training set randomly selected $\mathrm{k}$ objects, each object represents a cluster of initial average, also is at the center of clustering cluster. In the following formulas, we define this. 


$$
\begin{aligned}
& V=\left\{v_{1}, v_{2}, \ldots, v_{m}\right\} \\
& E=\sum_{i=1}^{k} \sum_{p \in C}\left|p-m_{i}\right|^{2}
\end{aligned}
$$

The theory of fuzzy technology involved in many applications in intrusion detection system, for IDS has opened up new areas of development but also to technology to be new challenges. Although fuzzy theory has been used to IDs of each function module, but town said that until recently has not been a real fuzzy IDs system $m$ now. Trust is a kind of trust is to be trusted expected and such expectations can have different degree measured by trust the trust degree. Trust can from complete distrust to fully trust. In the development process of host-based IDS became assimilated into other technology. Big data communication under the network stability evaluation index is more, generally take a direct correlation between each other is more effective. Obtained by fuzzy hierarchy analysis method under the different indexes of evaluation weights, collection of indicators presented in big data communication under the network stability analysis hierarchy, at the same time a large number of detection index and the fuzziness, different indexes can be obtained by fuzzy analytic.

To critical system files and executable files a commonly used method of intrusion detection, was conducted by regular inspection check and, in order to find surprising changes. Reaction speed and the frequency of the polling interval have a direct relationship. Many HIDS that works products are listening on port activities, and report to administrator when a specific port is access. This kind of test method will be based on the basic method of network intrusion detection into the detection on the basis of the host environment.

The Principles of Computer Network Robustness. Network stability refers to the network intrusion sudden crisis in the process, such as the size of the computer network anti-jamming, anti-destroying ability. The stability of the computer network can provide help for the sudden events in the large-scale data communication, to ensure the maximum benefits of time and the minimum network loss. This act big data in the user environment network stability evaluation need from two aspects of evaluation index, stability, and so on were analyzed. Network allows the user to free to release and get all kinds of information, so the network the threat is multifaceted. Can be transmission line of attack, also can make the network protocol, and hardware of computer software to carry out attacks. Usually, large-scale computer network itself has the characteristics of large scale and complicated structure, and the product, and there are some differences between each device to achieve reliable operation network, ensuring stability of information transmission, need through the use of advanced computer network management technology as support, however, the computer network construction and development process, there are computer management software is not reasonable, and not in accordance with the standard of a very good defensive role. However, the existing packet filtering technology can only be applied to the network layer.

Usually, large-scale computer network itself has the characteristics of large scale and complicated structure, and the product, and there are some differences between each device to achieve reliable operation network, ensuring stability of information transmission, need through the use of advanced computer network management technology as support, however, the computer network construction and development process, there are computer management software is not reasonable, and not in accordance with the standard of a very good defensive role. However, the existing packet filtering technology can only be applied to the network layer, couldn't filter for application layer packet. In the following figure, we show the fuzzy set based attack classification. 


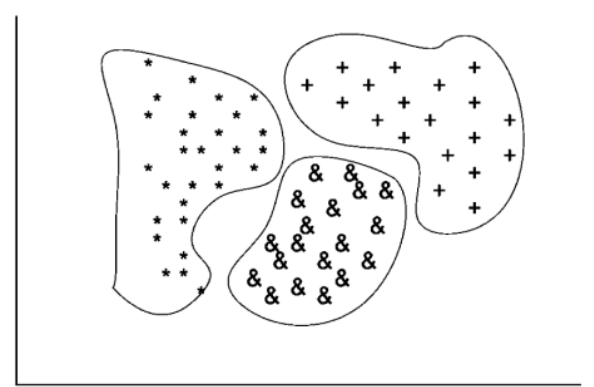

Fig. 2The Fuzzy Set based Attack Classification

Further improving computer network hardware and software, is an important way to improve stability and reliability of computer network. Computer network in the actual use process, magnetic radiation, line equipment damage, the influence of such factors will directly lead to leakage of the computer network information. In order to further solve the problem of the information leak, the relevant department can adopt the way of installation of the filter, or to install systemic radiation, electromagnetic field block and it can prevent illegal personnel for computer information intercepted. Computer viruses are constantly updated, to this end, regular well about computer virus software update is extremely important. Through the computer virus software updates and detection, can guarantee the security of computer network use. On the other hand, the use of firewall technology, through the use of firewall technology, can make the criminals cannot enter into the computer user's network, thus ensure the security of the computer user's information. In addition, in addition to the firewall technology, the use of data encryption, and improve the reliability of computer network which is also denoted as a common method of current computer users.

The Prospect and Future Research. With the popularization and development of computer network, the use of the network has penetrated into all fields. Dependent on the information society, people to network is also growing, but with the development of the network quickly, network security has already become a potentially huge problem. In the good times of network development, the network information security on a piece of dark cloud, although the computer industry has made great progress in our country, but its core components manufacturing technology is still very weak. Cloud security through the network of the end of the service software behavior anomaly detection of network, access to the Internet in the new information of malware, pushed to the server for automatic analysis and processing, a solution of viruses and again distributed to every customer service. Cloud security technology application, identification and killing the virus no longer rely solely on local hard drive virus, but rely on the huge network service, real-time collection, analysis and processing in real time. Cloud security concept, has caused widespread controversy, many people believe that it is false. But as companies use cloud security technology to achieve the concept of a new generation of killing, cloud security technology has become a fact of life. But at the same time, because of the cloud security is still a relatively new technology, so there are many problems need further exploration.

Along with the network all over the world, private network as demand also inevitably linked with external public sites directly or indirectly. Just due to network, the correlation of network attack one of the worst links in the chain can cause the collapse of the whole security system. More complicated so that the operation of the network environment, controllable sharply reduced, the network security also becomes poor. In actual use to evaluate security model, although the establishment of the model than the rules extraction is simple and can comprehensively reflect the security hidden danger existing in the system, but the existing research on the establishment of a safety assessment model because of the lack of subjective factors and model complete degree is easy to cause the uncertainty of the result. 


\section{CONCLUSIONS}

In this paper, we conduct research on the enhancement of computer network robustness methodology based on the fuzzy set theory. Computer network in the process of operation, due to the influence of many factors, its reliability, stability, study, work has made great influence to people, based on this, the computer network reliability become the most important work. This paper will, from the perspective of the computer network reliability concept and design principles, and factors affecting the reliability of the computer network are the thorough analysis and research, finally from the aspects of the network system in this paper, the computer network reliability is effective countermeasures.

\section{References}

[1] Sakakibara H, Kajitani Y, Okada N. Road Network Robustness for Avoiding Functional Isolation in Disasters[J]. Journal of Transportation Engineering, 2014, 130(5):560-567.

[2] Min B, Yi S D, Lee K M, et al. Network robustness of multiplex networks with interlayer degree correlations[J]. Physical Review E, 2014, 89(4):1600-1618.

[3] Chen S M, Zou X Q, Hui L, et al. Research on robustness of interdependent network for suppressing cascading failure[J]. Acta Physica Sinica, 2014, 63(2):257-264. 